\title{
The Effectiveness of GRADIOR:
}

\section{A Neuropsychological Rehabilitation Program for People with Mild Cognitive Impairment and Mild Dementia. Results of a Randomized Controlled Trial After 4 and 12 Months of Treatment}

\author{
Angie A. Diaz Baquero ${ }^{\mathrm{a}, \mathrm{e}, *}$, Manuel A. Franco-Martín ${ }^{\mathrm{b}, \mathrm{c}}$, Esther Parra Vidales ${ }^{\mathrm{d}}$, \\ José Miguel Toribio-Guzmán ${ }^{\mathrm{e}}$, Yolanda Bueno-Aguado ${ }^{\mathrm{f}}$, Fernando Martínez Abad ${ }^{\mathrm{g}}$,

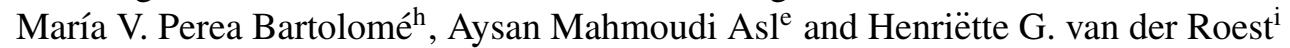 \\ ${ }^{a}$ Institute of Biomedical Research of Salamanca, University of Salamanca, Salamanca, Spain \\ ${ }^{\mathrm{b}}$ Department of Psychiatric, Rio Hortega University Hospital, Valladolid, Spain \\ ${ }^{\mathrm{c}}$ Department of Psychiatric, Zamora Healthcare Complex, Zamora, Spain \\ ${ }^{\mathrm{d}}$ IBIP Center for Clinical Care in Mental Health and Aging, INTRAS Foundation, Zamora, Spain \\ ${ }^{\mathrm{e}}$ Department of Research and Development, Iberico Institute for Research in Psychoscience (IBIP), INTRAS \\ Foundation, Zamora, Spain \\ ${ }^{\mathrm{f}}$ Department of Gradior and Cognitive Research, INTRAS Foundation, Valladolid, Spain \\ $\mathrm{g}$ University Institute of Educational Sciences, University of Salamanca, Salamanca, Spain \\ ${ }^{\mathrm{h}}$ Basic Psychology, Psychobiology and Methodology Department, Salamanca University, Salamanca, Spain \\ ${ }^{\mathrm{i}}$ Department on Aging, Netherlands Institute of Mental Health and Addiction (Trimbos Institute), Utrecht, \\ The Netherlands
}

Accepted 27 December 2021

Pre-press 3 February 2022

\begin{abstract}
.
Background: Computer-based cognitive training programs have been developed with promising results on the maintenance/improvement of cognitive performance in people with dementia.

Objective: The objective was to evaluate the effectiveness of the cognitive rehabilitation program "GRADIOR" in people with mild cognitive impairment and mild dementia.

Method: This study was a single-blind multicenter randomized clinical trial. Participants were recruited from hospitals/day centers. The experimental group (EG) and control group (CG) received computer-based cognitive training (CCT) and routine daily care, respectively. Outcome measures at $T_{0}$ : baseline, $T_{1}$ : at 4 months, $T_{2}$ : at 12 months were compared within and between-groups.
\end{abstract}

\footnotetext{
${ }^{*}$ Correspondence to: Angie A. Diaz Baquero, Institute of Biomedical Research of Salamanca, University of Salamanca, 37001
} 
Results: Significant differences or important effect sizes were detected at the intragroup and intergroup level for most variables, observing a trend of improvement and/or maintenance at 4 months by Visual Reasoning of Cambridge Cognitive Examination (CAMCOG), Digit and Arithmetic of WAIS-III, Semantic Verbal Fluency, Mini-Mental State Exam (MMSE), Trail Making Test (TMT)-A-Mistakes and at 12 months by Visual Reasoning of CAMCOG, Digit Symbol of WAIS-III, TMT-B-mistakes, Visual Memory of Rivermead Behavioural Memory Test, Lexical Verbal Fluency-P, Yesavage's Geriatric Depression Scale (GDS), TMT-A-time scales whose objective was to evaluate some executive functions and/or the memory. The CG presented a worsening trend for most of the measures towards 12 months. There was also a significant interaction between "time and group" for MMSE $\left(\mathrm{F}=8.971 ; p=0.03 ; \eta^{2}=0.019\right)$ and the GDS $\left(\mathrm{F}=3.414 ; p=0.04 ; \eta^{2}=0.041\right)$, as well as small effect sizes for TMT-A-time $\left(\mathrm{F}=1.641 ; p=0.21 ; \eta^{2}=0.021\right)$ and TMT-A-mistakes $\left(\mathrm{F}=0.908 ; p=0.41 ; \eta^{2}=0.019\right)$.

Conclusion: CCT with GRADIOR has been proved to benefit cognitive functions (ISRCTN:15742788).

Keywords: Cognitive training, dementia, mild cognitive impairment, randomized controlled trial, rehabilitation

\section{INTRODUCTION}

Dementia is a neurodegenerative disease that is characterized by a series of cognitive [1,2], emotional [3], physical [4], and social [5] deficits, which gain visibility as the disease progresses through its various stages. Mild cognitive impairment (MCI) has been regarded as an early stage of dementia, although it not always evolves in such direction. An individual with MCI could develop dementia if several risk factors converge [6, 7]. Hence, an early diagnosis of MCI is essential [8] to start therapy, which, although it cannot revert the symptoms, some of them can help preserve cognitive condition and/or delay cognitive decline.

People with dementia (PwD) can be treated with pharmacological therapy [9] and also with psychosocial therapies, the latter of which are aimed at physical, cognitive, social, and emotional, or even family unit rehabilitation [10].

One of the challenges of these psychosocial interventions is that they are difficult to implement, both because of their cost and because of the expertise these require. In this regard, new technologies offer the possibility of changing and improving such interventions so that these might be more accessible [11]. Examples of this are the e-Salud interventions, aimed at providing support for activities of daily living (ADL) $[12,13]$ or at improving the cognitive and emotional condition of PwD [14].

The improvements observed involve cognitive functions such as attention [15-17], memory [16, 18, 19] and executive function (EF) $[15,20]$ in people with MCI and dementia. Likewise, other studies have mentioned improvements in social and emotional aspects, such as anxiety levels [15] and depression [21].
Although the scientific literature includes a broad variety of studies on the effectiveness of computerbased cognitive training (CCT) programs [21, 22], it is necessary to strengthen the evidence base of the usefulness of this type of intervention. The lack of methodological rigor associated with limitations such as sample size [23] and short CCT periods $[24,25]$ hinders the gathering of solid evidence [26]. Therefore, it is necessary to further assess the "effectiveness" of these interventions using randomized controlled trials (RCT).

The aim of this study is to evaluate the effectiveness of a CCT program (GRADIOR) on cognition and emotional condition in people with MCI and mild dementia by conducting an RCT [27]. This study will only present the effectiveness results associated with the GRADIOR intervention up to 4 and 12 months. The follow-up period at 16 and 24 months, as well as the results associated with some secondary measures, will be published later.

\section{METHODS}

\section{Study design}

The design used was a simple-blind, multicenter, RCT (ISRCTN:15742788) [28]. Our design initially included four parallel groups [27]. However, the development of the ehcoBUTLER platform was initially part of a European project that failed to become ready for our RCT. Therefore, we decided not to include it. This led to a change in the number of parallel groups, the allocation ratio, and the sample size.

The sample population was recruited between June 2018 and December 2019. The participants 


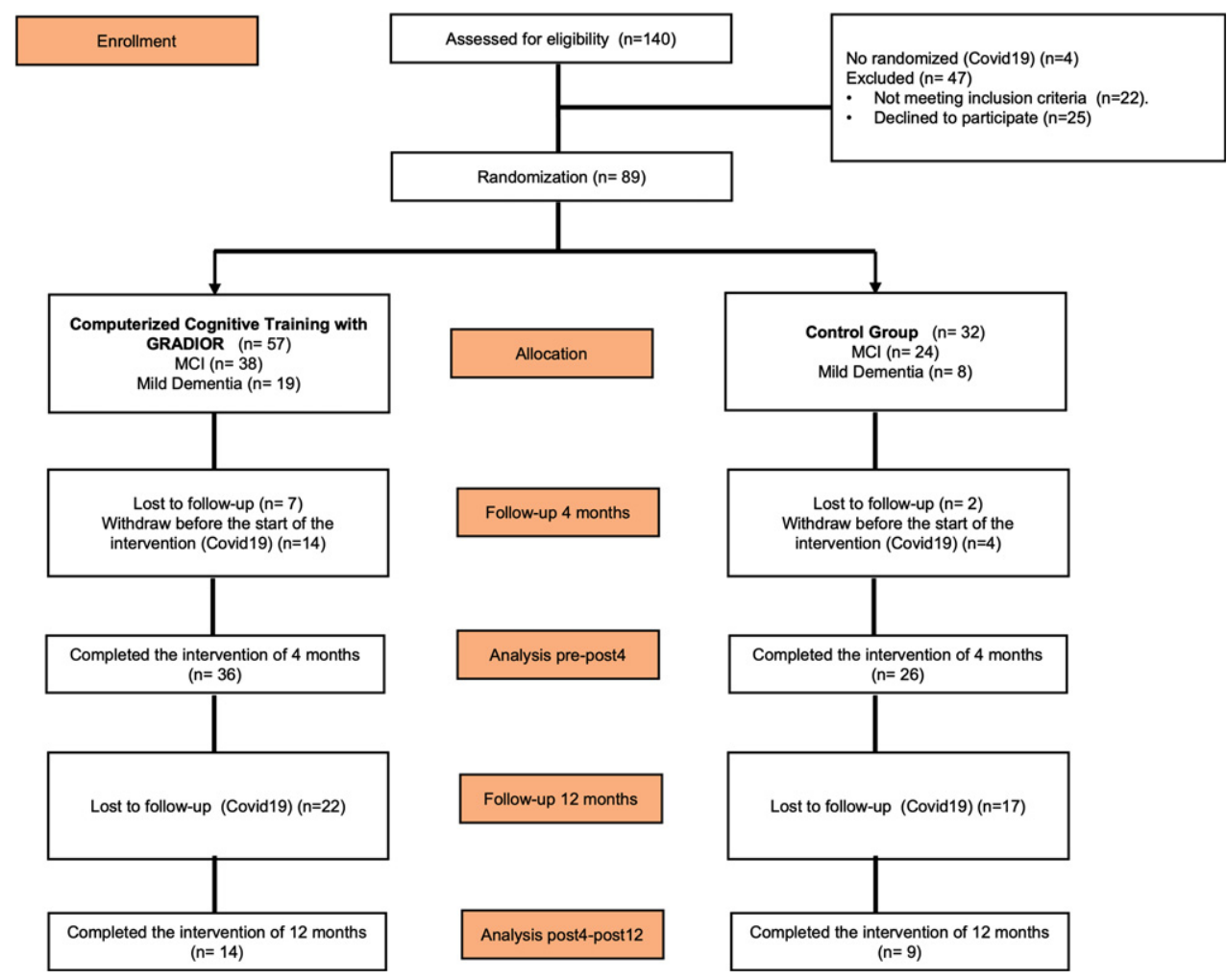

Fig. 1. Recruitment and randomization process.

were randomized (1:2) to one of the two study groups by a simple random assignment (Fig. 1). We used a computer-generated random number sequence through the Epidat 4.1 program. An independent researcher generated the assignment. The evaluators were neuropsychologists who were blinded. The inter-rater reliability was $(\alpha=99.1 \%)$. The therapists who supervised each session and the participants were not blinded. Participants agreed to participate prior to randomization and without knowing which group they would be assigned. The individuals in the experimental group (EG) attended two or three weekly CCT sessions (depended on each center) using GRADIOR over a 12-month period, whereas those in the control group (CG) received usual care.

This study was multicenter because it involved daycare centers, memory clinics, and hospitals in the Spanish regions of Castile and León, and Galicia. The study was approved on May 17, 2017, by the Medication Research Ethics Committee of healthcare area of Zamora (Number:387-E.C). Informed consent was obtained from all the participants and caregivers.

\section{Participants}

Eighty-nine people participated in this study. These were randomly assigned to the experimental $(n=57)$ and control $(n=32)$ group. The dropout rate for this study before 4 months was $10 \%$, although $20 \%$ of the participants did not start the intervention due to the onset of the COVID-19 pandemic. The final sample consisted of 62 participants who completed 4-months of intervention. Only 23 participants completed the scheduled 12 months, which could not be completed by the rest of participants because of the outbreak of the COVID-19 pandemic (Fig. 1).

Participants were aged 60-90 and were clinically diagnosed with MCI according to Petersen [29] and with mild dementia according to the Diagnostic and Statistical Manual of Mental Disorders (DSM-V) [30]. A psychogeriatrician and neurologist made the diagnosis according to the criteria mentioned above and considering the patient's medical history. The MCI types were amnestic, and types of dementia included were as follows: Alzheimer's disease, vascular dementia, mixed and frontotemporal dementia. Scores obtained on the Mini-Mental State Exam 
(MMSE) were additional: MMSE $\leq 27$ for MCI, and MMSE: $20 \leq \mathrm{x} \leq 25$ for mild dementia, adjusting each score according to the participant's age and level of education [31]. A score of $\leq 5$ on Yesavage's Geriatric Depression Scale (GDS) was required, each participant should participate voluntarily, have a reference caregiver, and be fluent in speaking and understanding Spanish.

On the other hand, the participants were excluded when they had severe physical comorbidities, significant sensory disturbances (hearing-visual), neurological disorders (Huntington's disease, traumatic brain injury, Parkinson's disease), clinically significant psychopathological disorders (depression, anxiety, bipolar disorder, psychosis), and/or a history of psychoactive substance use (alcohol, tobacco).

\section{Neuropsychological assessment}

The participants of both groups were assessed at three different moments throughout the intervention $\left(\mathrm{T}_{0}\right.$ : at baseline, $\mathrm{T}_{1}$ : at 4 months, and at $\mathrm{T}_{2}: 12$ months of intervention). The cognitive scales used for the main outcome measurements were the MMSE [32], the cognitive subscale of the Alzheimer's Disease Assessment Scale [33], TMT-A-B [34], the Clock Drawing Test [35], Digit Symbol, Arithmetic and Digits of the Wechsler Adult Intelligence Scale (WAIS-III) [36], Visual Memory of the Rivermead Behavioural Memory Test (RBMT) [37], Visual Reasoning of the Cambridge Cognitive Examination (CAMCOG) [38], and Verbal Fluency Test [39, 40]. Secondary result measurements were associated with GDS [41]. These instruments have been described in greater detail in the protocol published on the RCT [27].

\section{Computer-based cognitive training with GRADIOR}

GRADIOR is a computer-based cognitive training program. It is a program that provides good usability and user experience [42-49]. This program includes a wealth of orientation, memory, attention, perception, $\mathrm{EF}$, reasoning, and calculation exercises. It allows the design and customization of intervention plans according to the type and level of the patient's cognitive disorder $[45,50]$. The cognitive intervention plan for this RCT was designed according to the cognitive profile (Supplementary Figure 1) and the level of difficulty for each exercise was adjusted to the cognitive level of each people. The participants attended two or three weekly 30 -min sessions over a 12-month period. The sessions were carried out in specialized rooms with computers in daycare centers, memory clinics, and hospitals.

\section{Statistical analysis}

The statistical analysis was conducted using the Statistical Package for the Social Sciences software package (SPSS) [51]. The Shapiro-Wilk Test of normality was used, taking sample size into account to identify the type of distribution of each of the variables. The comparison between the EG-CG was performed using the Mann-Whitney U Test for two independent samples to check whether there were significant differences between both groups regarding at each of the times $\mathrm{T}_{0}-\mathrm{T}_{1}-\mathrm{T}_{2}$ (intergroup). The Wilcoxon signed-Rank Test was used to compare the cognitive performance between times $T_{0}-T_{1}$ and $\mathrm{T}_{1}-\mathrm{T}_{2}$ for each of the groups, which allowed the identification of changes over time (intragroup). The significance threshold set for each of the analyses was $\leq 0.05$.

To run the repeated measures ANOVA Test, it was necessary to verify the assumptions of homoscedasticity $(p \geq 0.05)$ and sphericity $(p \geq 0.05)$. The Greenhouse-Geisser correction was used in those cases where homoscedasticity was not met. The assumption of sphericity was tested using Mauchly's Test of Sphericity. The repeated measures ANOVA analysis allowed us to see the time factor (changes over time in the sample in general) and the interaction between factors "time versus study group" (differences between the two groups over time) and "time, clinical group, and study group" (differences over time with respect to the study group and the clinical group).

\section{RESULTS}

\section{Sample characteristics}

The sample that completed 4 months of intervention consisted of 62 individuals. The mean age was $74.73 \pm 6.63$ and $27.4 \%$ were men. The mean number of years of education was $9.36 \pm 2.68$, and $66.1 \%$ $(n=41)$ of the individuals had a diagnosis of MCI, and $33.9 \%(n=21)$ of mild dementia. The final sample comprised a total of 23 individuals who completed the 12 months of CCT. Of these, $26.1 \%$ were men. The mean age was $76.30 \pm 7.69$ and the mean number of years of education was $9.96 \pm 3.11$. Of the sample, 
$60.9 \%(n=14)$ and $39.1 \%(n=9)$ of the individuals were diagnosed with MCI and with mild dementia, respectively. However, there were no significant differences between EG-CG regarding age, years of education, sex, or clinic group at 4 or 12 months (Table 1).

\section{Comparison between groups for each condition} $\left(T_{0}-T_{1}-T_{2}\right)$ (intergroup)

The Shapiro-Wilk Test revealed that most of the variables were not normally distributed, which led to the use of the Mann-Whitney U Test. There were no significant differences between the two groups as to $T_{0}, T_{1}$, and $T_{2}$. However, a medium-moderate effect size $(d=0.460)$ was found for Visual Reasoning of CAMCOG in $\mathrm{T}_{1}(n=23)$, the EG performing better than the CG $(2.07 \pm 1.14$ and $2.00 \pm 1.50$, respectively). Small effect sizes in $\mathrm{T}_{1}$ were found for TMT-B-Time $(\mathrm{d}=0.317)$ and Arithmetic of WAISIII $(\mathrm{d}=-0.302)$, and in $\mathrm{T}_{2}$ for Visual Memory of RBMT $(\mathrm{d}=-0.317)$ and Digit Symbol of WAIS-III $(\mathrm{d}=0.302)$ (Table 2).

\section{Evolution of cognitive-psychological} performance for each group (intragroup)

The Shapiro-Wilk Test revealed that most of the variables were not normally distributed for either group during $\mathrm{T}_{0}-\mathrm{T}_{1}$ and $\mathrm{T}_{1}-\mathrm{T}_{2}$. Based on this and on sample size within each group, the Wilcoxon signedrank Test was conducted (Table 3 ).

Regarding the changes seen between $T_{0}$ and $T_{1}$ for EG, we highlight an improvement trend of EG during $\mathrm{T}_{1}$. In this way, we find a small effect sizes for Visual Reasoning of CAMCOG $(\mathrm{d}=-0.172)$, WAIS-III Digits $(\mathrm{d}=-0.123)$, WAIS-III Arithmetic $(\mathrm{d}=-0.244)$, and Semantic Verbal Fluency (SVF) $(\mathrm{d}=-0.273)$, and medium-moderate for Lexical Verbal Fluency M $(\mathrm{d}=-0.310)$, MMSE $(\mathrm{d}=-0.439)$, and TMT-AMistakes $(\mathrm{d}=0.436)$. However, a worsening trend was only detected in TMT-B-Time with a mediummoderate effect size $(d=0.362)$

On the other hand, we observed that for the $\mathrm{T}_{1}-\mathrm{T}_{2}$ condition in $\mathrm{EG}$, there was a trend of improvement with small effect sizes for CAMCOGVisual Reasoning $(\mathrm{d}=-0.111)$ and WAIS-III Digit Symbol $(d=-0.200)$, medium-moderate for TMTB-mistakes $(\mathrm{d}=0.359)$ and RBMT-Visual Memory $(d=-0.364)$, moderate for Lexical Verbal Fluency $($ LVF-P) $(\mathrm{d}=-0.489)$ and GDS $(\mathrm{d}=0.538)$, and there were significant differences $(p=0.02)$ and a large

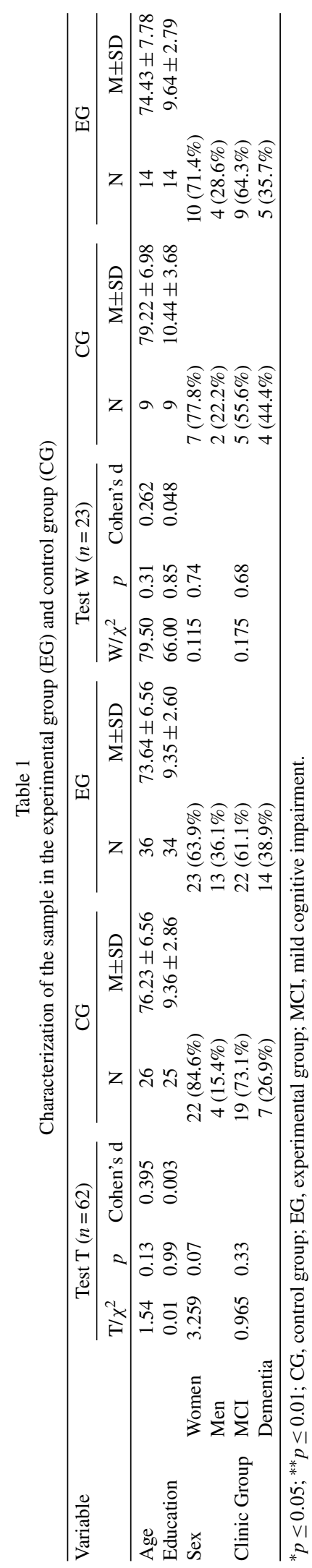


Table 2

Mann-Whitney $\mathrm{U}$ test. Intergroup comparison (EG and CG) during each time $\left(\mathrm{T}_{0}, \mathrm{~T}_{1}, \mathrm{~T}_{2}\right)$

\begin{tabular}{|c|c|c|c|c|c|c|c|c|c|c|c|c|}
\hline \multirow[t]{3}{*}{ Test } & \multicolumn{3}{|c|}{$\mathrm{T}_{0}$} & \multicolumn{3}{|c|}{$\mathrm{T}_{1}$} & \multicolumn{3}{|c|}{$\mathrm{T}_{1}$} & \multicolumn{3}{|c|}{$\mathrm{T}_{2}$} \\
\hline & \multicolumn{6}{|c|}{$N=62$} & \multicolumn{6}{|c|}{$N=23$} \\
\hline & $\mathrm{W}$ & $p$ & Cohen's d & $\mathrm{W}$ & $p$ & Cohen's d & W & $p$ & Cohen's d & $\mathrm{W}$ & $p$ & Cohen's d \\
\hline MMSE & 509 & 0.56 & 0.088 & 482 & 0.85 & 0.030 & 56.500 & 0.70 & -0.103 & 69.000 & 0.73 & 0.095 \\
\hline ADAS-Cog & 456 & 0.87 & -0.026 & 425.5 & 0.55 & -0.091 & 66.000 & 0.87 & 0.048 & 66.000 & 0.87 & 0.048 \\
\hline TMT A Time & 400 & 0.28 & -0.145 & 527 & 0.37 & 0.126 & 73.500 & 0.41 & 0.167 & 69.000 & 0.71 & 0.095 \\
\hline TMT-A Mistakes & 480 & 0.83 & 0.026 & 522 & 0.33 & 0.115 & 70.500 & 0.65 & 0.119 & 68.000 & 0.67 & 0.079 \\
\hline TMT B Time & 456.5 & 0.85 & -0.025 & 485.5 & 0.78 & 0.037 & 83.000 & 0.16 & 0.317 & 76.000 & 0.33 & 0.206 \\
\hline TMT-B Mistakes & 477.5 & 0.90 & 0.020 & 414.5 & 0.44 & -0.114 & 64.000 & 0.97 & 0.016 & 72.000 & 0.59 & 0.143 \\
\hline WAIS-III: Digits & 483.5 & 0.83 & 0.033 & 416.5 & 0.46 & -0.110 & 79.000 & 0.32 & 0.254 & 58.500 & 0.80 & -0.071 \\
\hline WAIS-III: Digit Symbol & 540 & 0.30 & 0.154 & 481 & 0.85 & 0.028 & 59.000 & 0.82 & -0.063 & 82.000 & 0.24 & 0.302 \\
\hline WAIS III: Arithmetic & 553 & 0.22 & 0.182 & 501 & 0.38 & 0.133 & 44.000 & 0.24 & -0.302 & 67.000 & 0.82 & 0.063 \\
\hline CAMCOG: Visual Reasoning & 428 & 0.56 & -0.085 & 538.5 & 0.31 & 0.151 & 92.000 & 0.07 & 0.460 & 52.000 & 0.50 & -0.175 \\
\hline RBMT: Visual Memory & 396 & 0.30 & -0.154 & 462.5 & 0.94 & -0.012 & 63.500 & 1.000 & 0.008 & 43.000 & 0.20 & -0.317 \\
\hline CDT Order & 451.5 & 0.97 & -0.008 & 552.5 & 0.22 & 0.181 & 79.500 & 0.29 & 0.262 & 56.000 & 0.68 & -0.111 \\
\hline CDT Copy & 473.5 & 0.94 & 0.012 & 468.5 & 0.70 & 0.060 & 74.500 & 0.48 & 0.183 & 68.500 & 0.74 & 0.087 \\
\hline SVF & 510.5 & 0.31 & 0.155 & 482 & 0.55 & 0.090 & 50.500 & 0.83 & -0.065 & 54.500 & 0.61 & -0.135 \\
\hline LVF-P & 478.5 & 0.59 & 0.083 & 528.5 & 0.19 & 0.196 & 65.000 & 0.45 & 0.204 & 62.500 & 1.000 & -0.008 \\
\hline LVF-M & 474.5 & 0.63 & 0.074 & 427 & 0.56 & -0.088 & 62.000 & 0.59 & 0.148 & 73.000 & 0.54 & 0.159 \\
\hline LVF-R & 502.5 & 0.37 & 0.137 & 482 & 0.85 & 0.030 & 52.500 & 0.94 & -0.028 & 53.000 & 0.55 & -0.159 \\
\hline GDS & 444 & 0.74 & -0.051 & 425.5 & 0.55 & -0.091 & 58.000 & 0.77 & -0.079 & 74.000 & 0.50 & 0.175 \\
\hline
\end{tabular}

${ }^{*} p \leq 0.05 ;{ }^{* *} p \leq 0.01$; ADASCog, Alzheimer's disease Assessment Scale: cognitive subscale; CAMCOG, Cambridge Cognition Examination; CDT, Clock Drawing Test; GDS, Geriatric Depression Scale; LVF, Lexical Verbal Fluency; MMSE, Mini-Mental State Examination; RBMT, Rivermead Behavioural Memory Test; SVF, Semantic Verbal Fluency; $\mathrm{T}_{0}$, baseline; $\mathrm{T}_{1}$, at 4 months of intervention; $\mathrm{T}_{2}$, at 12 months of intervention; TMT, Trail Making Test; WAIS-III, Wechsler Adult Intelligence Scale.

effect size $(\mathrm{d}=-1000)$ for TMT-A-time. However, slight worsening tendencies were also detected for the WAIS-III Arithmetic with moderate effect sizes $(d=0.636)$ and, a high effect size with a significant difference for the MMSE $(p=0.01 ; \mathrm{d}=0.868)$ and LVF-M $(p=0.03 ; \mathrm{d}=1000)$.

Regarding the CG, this exhibited an improvement trend during $\mathrm{T}_{1}$ and had small effect sizes for Digit Symbol (WAIS-III) $(\mathrm{d}=-0.281)$, LVFM $(\mathrm{d}=-0.289), \quad$ LVF-R $(\mathrm{d}=-0.294)$, WAIS-III Arithmetic $(\mathrm{d}=-0.327)$, medium-moderate for TMTB-mistakes $(\mathrm{d}=0.382)$, moderate for TMT-A-Time $(\mathrm{d}=-0.552)$ and WAIS-III Digits $(\mathrm{d}=-0.636)$, this last variable with significant differences $(p=0.01)$. And this group showed a worsening tendency for TMT-A-mistakes $(\mathrm{d}=-0.258)$.

On the other hand, the CG also showed an improvement trend in $\mathrm{T}_{2}$ with moderate effect sizes for RBMT-Visual Memory $(\mathrm{d}=-0.500)$, medium-high for TMT-A-mistakes $(\mathrm{d}=0.700)$, and a large for TMT-B-Time $(\mathrm{d}=-1000)$. However, CG showed a worsening trend during $\mathrm{T}_{2}$ with small effect sizes for WAIS-III Digit Symbol $(d=0.200)$, LVF-M ( $d=0.267)$, and GDS $(\mathrm{d}=-0.286)$, mediummoderate for SVF $(d=0.429)$, moderate for CAMCOG-Visual Reasoning ( $\mathrm{d}=0.500)$, WAIS-III Arithmetic $(\mathrm{d}=0.583)$, and Clock Drawing Test
(CDT)-Copy $(\mathrm{d}=0.600)$, medium-high for WAIS-III Digits $(\mathrm{d}=0.694)$ and CDT-Order $(\mathrm{d}=0.667)$.

\section{Repeated measures ANOVA}

Most of the variables met the assumption of homoscedasticity. For the cases in which this principle was not fulfilled, we applied the GreenhouseGeisser correction [52]. Thus, variance in the $\mathrm{CG}$ and in the EG in each of the variables for $\mathrm{T}_{0}-\mathrm{T}_{1}-\mathrm{T}_{2}$ was the same. Most of the variables also met the assumption of sphericity, except for AdasCog-total, TMT-B-Time-Mistakes, and WAISIII Digit-Symbol. After running the repeated measures ANOVA, the effect of the time factor $\left(\mathrm{T}_{0}\right.$, $\mathrm{T}_{1}, \mathrm{~T}_{2}$ ) was significant on the cognitive performance associated with the tests: Digits $(\mathrm{F}=3.632 ; p=0.04$; $\left.\eta^{2}=0.030\right)$ and Arithmetic of WAIS-III $(\mathrm{F}=4.372$; $p=0.02 ; \eta^{2}=0.038$ ) (Table 4). Therefore, cognitive performance was not the same in the three times recorded and we identified an increase in cognitive performance at 4 months and a slight decrease at 12 months in the general sample. Although, we can say that they remained around the average, so their performance was not low (Fig. 2).

There was a statistically significant interaction between time and study group (experimental and 
Table 3

Wilcoxon signed-rank test. Intragroup comparison (EG-CG) with respect to cognitive performance between $\mathrm{T}_{0}-\mathrm{T}_{1}$ and $\mathrm{T}_{1}-\mathrm{T}_{2}$

\begin{tabular}{|c|c|c|c|c|c|c|c|c|c|c|c|c|c|}
\hline \multirow[t]{3}{*}{ Test } & \multirow[t]{3}{*}{ Group } & \multirow[t]{3}{*}{$p$} & \multirow[t]{3}{*}{ Cohen's d } & \multicolumn{2}{|r|}{$\mathrm{T}_{0}$} & \multicolumn{2}{|r|}{$\mathrm{T}_{1}$} & \multirow[t]{3}{*}{$p$} & \multirow[t]{3}{*}{ Cohen's d } & \multicolumn{2}{|r|}{$\mathrm{T}_{1}$} & \multicolumn{2}{|r|}{$\mathrm{T}_{2}$} \\
\hline & & & & \multicolumn{4}{|c|}{$\overline{(n}=6 \overline{2)}$} & & & \multicolumn{4}{|c|}{$(n=23)$} \\
\hline & & & & $\mathrm{N}$ & $\bar{x} \pm \mathrm{SD}$ & $\mathrm{N}$ & $\bar{x} \pm \mathrm{SD}$ & & & $\mathrm{N}$ & $\bar{x} \pm \mathrm{SD}$ & $\mathrm{N}$ & $\bar{x} \pm \mathrm{SD}$ \\
\hline \multirow[t]{2}{*}{ MMSE } & Control & 0.20 & -0.337 & 26 & $24.15 \pm 4.16$ & 26 & $24.81 \pm 4.06$ & 1.000 & -0.048 & 9 & $24.11 \pm 4.46$ & 9 & $24.22 \pm 4.97$ \\
\hline & Experimental & $0.05^{*}$ & -0.439 & 36 & $24.28 \pm 2.54$ & 36 & $24.97 \pm 2.91$ & $0.01^{* *}$ & 0.868 & 14 & $25.21 \pm 2.33$ & 14 & $23.43 \pm 2.56$ \\
\hline \multirow[t]{2}{*}{ ADAS-Cog } & Control & 0.58 & 0.134 & 26 & $14.88 \pm 7.75$ & 26 & $14.31 \pm 7.29$ & 0.57 & 0.250 & 9 & $17.11 \pm 10.52$ & 9 & $16.44 \pm 13.14$ \\
\hline & Experimental & 0.62 & -0.103 & 35 & $14.00 \pm 4.99$ & 35 & $14.43 \pm 6.48$ & 0.40 & -0.275 & 13 & $12.69 \pm 4.03$ & 14 & $14.29 \pm 4.01$ \\
\hline \multirow[t]{2}{*}{ TMT A Time } & Control & 0.07 & -0.552 & 25 & $7.80 \pm 5.61$ & 22 & $9.09 \pm 6.66$ & 0.89 & -0.133 & 7 & $10.71 \pm 10.97$ & 8 & $13.75 \pm 14.33$ \\
\hline & Experimental & 1.00 & -0.007 & 33 & $11.21 \pm 11.32$ & 35 & $13.43 \pm 16.21$ & $0.03^{*}$ & -1.000 & 14 & $9.29 \pm 10.72$ & 14 & $11.79 \pm 11.87$ \\
\hline \multirow[t]{2}{*}{ TMT-A Mistakes } & Control & 0.47 & -0.258 & 26 & $0.54 \pm 1.07$ & 25 & $0.64 \pm 1.19$ & 0.27 & 0.700 & 9 & $0.89 \pm 1.54$ & 9 & $0.22 \pm 0.44$ \\
\hline & Experimental & 0.17 & 0.436 & 36 & $0.42 \pm 0.77$ & 36 & $0.25 \pm 0.44$ & 1.000 & - & 14 & $0.21 \pm 0.43$ & 14 & $0.14 \pm 0.36$ \\
\hline \multirow[t]{2}{*}{ TMT B Time } & Control & 0.79 & 0.106 & 9 & $14.44 \pm 11.02$ & 10 & $15.00 \pm 9.13$ & 0.37 & -1.000 & 3 & $15.00 \pm 13.23$ & 2 & $25.00 \pm 7.07$ \\
\hline & Experimental & 0.25 & 0.362 & 11 & $15.91 \pm 18.68$ & 15 & $15.33 \pm 11.26$ & 1.000 & 0.333 & 5 & $14.00 \pm 9.62$ & 5 & $10.00 \pm 6.12$ \\
\hline \multirow[t]{2}{*}{ TMT-B Mistakes } & Control & 0.19 & 0.382 & 17 & $1.59 \pm 1.42$ & 20 & $1.40 \pm 1.31$ & 0.53 & 0.333 & 5 & $1.20 \pm 1.79$ & 7 & $2.14 \pm 1.77$ \\
\hline & Experimental & 0.34 & 0.214 & 28 & $2.14 \pm 1.46$ & 31 & $2.23 \pm 1.52$ & 0.29 & 0.359 & 12 & $2.25 \pm 1.66$ & 12 & $1.83 \pm 1.53$ \\
\hline \multirow[t]{2}{*}{ WAIS-III: Digits } & Control & $0.01^{* *}$ & -0.636 & 26 & $10.65 \pm 2.17$ & 25 & $11.48 \pm 2.20$ & 0.09 & 0.694 & 9 & $11.44 \pm 2.46$ & 9 & $10.11 \pm 3.14$ \\
\hline & Experimental & 0.59 & -0.123 & 36 & $10.47 \pm 2.61$ & 36 & $10.58 \pm 2.95$ & 0.96 & 0.036 & 14 & $10.57 \pm 2.14$ & 14 & $10.57 \pm 2.74$ \\
\hline \multirow[t]{2}{*}{ WAIS-III: Digit Symbol } & Control & 0.25 & -0.281 & 23 & $10.17 \pm 2.06$ & 23 & $10.35 \pm 2.21$ & 0.85 & 0.200 & 8 & $11.00 \pm 2.45$ & 8 & $10.75 \pm 2.66$ \\
\hline & Experimental & 0.76 & -0.081 & 36 & $9.83 \pm 2.87$ & 35 & $10.11 \pm 2.41$ & 0.62 & -0.200 & 14 & $9.57 \pm 2.28$ & 14 & $9.79 \pm 1.72$ \\
\hline \multirow[t]{2}{*}{ WAIS III: Arithmetic } & Control & 0.24 & -0.327 & 26 & $10.50 \pm 2.34$ & 25 & $10.68 \pm 2.67$ & 0.15 & 0.583 & 9 & $10.44 \pm 2.92$ & 9 & $9.00 \pm 3.74$ \\
\hline & Experimental & 0.25 & -0.244 & 36 & $9.75 \pm 3.08$ & 36 & $10.22 \pm 2.82$ & 0.08 & 0.636 & 14 & $10.36 \pm 2.10$ & 14 & $9.00 \pm 3.11$ \\
\hline \multirow[t]{2}{*}{ CAMCOG: Visual Reasoning } & Control & 0.75 & -0.100 & 26 & $2.00 \pm 1.10$ & 25 & $1.96 \pm 1.06$ & 0.59 & 0.500 & 9 & $2.00 \pm 1.50$ & 9 & $1.78 \pm 1.56$ \\
\hline & Experimental & 0.45 & -0.172 & 36 & $2.22 \pm 1.42$ & 35 & $2.43 \pm 1.20$ & 0.80 & -0.111 & 14 & $2.07 \pm 1.14$ & 14 & $2.14 \pm 1.29$ \\
\hline \multirow[t]{2}{*}{ RBMT. Visual Memory } & Control & 0.84 & -0.058 & 26 & $7.00 \pm 3.02$ & 25 & $6.84 \pm 3.01$ & 0.26 & -0.500 & 9 & $6.11 \pm 3.18$ & 9 & $6.78 \pm 3.03$ \\
\hline & Experimental & 0.70 & 0.089 & 36 & $7.94 \pm 2.10$ & 36 & $7.78 \pm 2.38$ & 0.33 & -0.364 & 14 & $7.71 \pm 2.09$ & 14 & $8.43 \pm 2.41$ \\
\hline CDT-Order & Control & 0.40 & -0.212 & 26 & $7.25 \pm 2.74$ & 25 & $7.56 \pm 2.07$ & 0.22 & 0.667 & 9 & $7.56 \pm .2 .78$ & 9 & $6.72 \pm 3.09$ \\
\hline & Experimental & 0.62 & -0.117 & 34 & $7.35 \pm 2.24$ & 36 & $7.60 \pm 2.38$ & 0.88 & -0.073 & 14 & $7.57 \pm 2.70$ & 14 & $7.64 \pm 2.06$ \\
\hline CDT-Copy & Control & 0.81 & -0.068 & 26 & $8.81 \pm 1.86$ & 25 & $8.76 \pm 1.93$ & 0.35 & 0.600 & 9 & $8.44 \pm 2.86$ & 9 & $8.00 \pm 3.43$ \\
\hline & Experimental & 0.58 & 0.133 & 36 & $9.10 \pm 1.14$ & 36 & $8.99 \pm 1.50$ & 0.88 & 0.073 & 14 & $9.00 \pm 1.51$ & 14 & $9.00 \pm 1.00$ \\
\hline SVF & Control & 1.00 & 0.000 & 25 & $7.80 \pm 3.28$ & 25 & $7.56 \pm 3.37$ & 0.37 & 0.429 & 9 & $7.67 \pm 3.97$ & 9 & $7.33 \pm 4.00$ \\
\hline & Experimental & 0.21 & -0.273 & 34 & $7.03 \pm 2.88$ & 34 & $7.62 \pm 3.59$ & 0.80 & 0.109 & 12 & $8.75 \pm 3.86$ & 14 & $8.43 \pm 2.82$ \\
\hline LVF-P & Control & 0.51 & -0.162 & 25 & $7.72 \pm 3.55$ & 25 & $8.16 \pm 3.22$ & 1.000 & -0.067 & 9 & $8.00 \pm 4.30$ & 9 & $7.67 \pm 4.12$ \\
\hline & Experimental & 0.55 & -0.129 & 34 & $7.56 \pm 3.04$ & 34 & $7.82 \pm 2.76$ & 0.21 & -0.489 & 12 & $7.33 \pm 2.77$ & 14 & $8.29 \pm 3.02$ \\
\hline LVF-M & Control & 0.24 & -0.289 & 25 & $7.48 \pm 3.66$ & 25 & $8.28 \pm 3.69$ & 0.68 & 0.267 & 9 & $8.78 \pm 4.27$ & 9 & $8.44 \pm 3.88$ \\
\hline & Experimental & 0.18 & -0.310 & 34 & $7.68 \pm 3.44$ & 34 & $8.26 \pm 3.42$ & $0.03^{*}$ & 1.000 & 12 & $8.25 \pm 4.11$ & 14 & $7.71 \pm 4.03$ \\
\hline LVF-R & Control & 0.24 & -0.294 & 25 & $8.84 \pm 2.53$ & 25 & $9.40 \pm 2.90$ & 0.78 & 0.200 & 9 & $8.89 \pm 3.66$ & 9 & $8.00 \pm 3.39$ \\
\hline & Experimental & 0.38 & -0.200 & 34 & $8.50 \pm 2.57$ & 34 & $8.97 \pm 2.58$ & 0.89 & 0.061 & 12 & $8.83 \pm 2.52$ & 14 & $8.93 \pm 2.92$ \\
\hline GDS & Control & 0.69 & -0.105 & 26 & $3.42 \pm 3.23$ & 25 & $3.32 \pm 3.09$ & 0.59 & -0.286 & 9 & $2.89 \pm 2.20$ & 9 & $3.22 \pm 2.17$ \\
\hline & Experimental & 0.49 & -0.153 & 36 & $3.86 \pm 3.66$ & 36 & $4.19 \pm 3.81$ & 0.10 & 0.538 & 14 & $4.14 \pm 3.98$ & 14 & $3.07 \pm 3.27$ \\
\hline
\end{tabular}

${ }^{*} p \leq 0.05 ;{ }^{* *} p \leq 0.01$; ADASCog, Alzheimer's disease Assessment Scale: cognitive subscale; CAMCOG, Cambridge Cognition Examination; CDT, Clock Drawing Test; GDS, Geriatric Depression Scale; LVF, Lexical Verbal Fluency; MMSE, Mini-Mental State Examination; RBMT, Rivermead Behavioural Memory Test; SVF, Semantic Verbal Fluency; TMT, Trail Making Test; T 0 , baseline;

$\mathrm{T}_{1}$, at 4 months of intervention; $\mathrm{T}_{2}$, at 12 months of intervention; WAIS-III, Wechsler Adult Intelligence Scale. 
Table 4

Levene's test, sphericity, and ANOVA. Comparison of cognitive performance between $\mathrm{T}_{0}, \mathrm{~T}_{1}-\mathrm{T}_{2}(n=23)$

\begin{tabular}{|c|c|c|c|c|c|c|c|c|c|c|c|c|c|c|}
\hline \multirow[t]{3}{*}{ Test } & \multicolumn{6}{|c|}{ LEVENE'S TEST } & \multirow{2}{*}{\multicolumn{2}{|c|}{ SPHERICITY }} & \multicolumn{6}{|c|}{ ANOVA } \\
\hline & \multicolumn{2}{|c|}{$\mathrm{T}_{0}$} & \multicolumn{2}{|c|}{$\mathrm{T}_{1}$} & \multicolumn{2}{|c|}{$\mathrm{T}_{2}$} & & & \multicolumn{3}{|c|}{$\mathrm{T}_{0}-\mathrm{T}_{1}-\mathrm{T}_{2}$} & \multicolumn{3}{|c|}{$\mathrm{T}_{0}-\mathrm{T}_{2}$ Group } \\
\hline & $\mathrm{F}$ & $p$ & $\mathrm{~F}$ & $p$ & $\mathrm{~F}$ & $p$ & $\chi^{2}$ & $p$ & $\mathrm{~F}$ & $p$ & $\eta^{2}$ & $\mathrm{~F}$ & $p$ & $\eta^{2}$ \\
\hline MMSE & 22.402 & $<0.00$ & 5.943 & 0.02 & 8.983 & 0.01 & 4.965 & $0.08^{*}$ & 2.165 & 0.13 & 0.011 & 8.971 & $0.03^{*}$ & 0.019 \\
\hline ADAS-Cog & 2.543 & $0.13^{*}$ & 2.562 & $0.12^{*}$ & 13.533 & 0.00 & 152.552 & $<0.00$ & 0.630 & 0.44 & 0.010 & 0.610 & 0.44 & 0.009 \\
\hline TMT A Time & 0.402 & $0.55^{*}$ & 27.517 & $<0.00$ & 7.713 & 0.01 & 5.567 & $0.06^{*}$ & 0.532 & 0.59 & 0.007 & 1.641 & 0.21 & 0.021 \\
\hline TMT-A Mistakes & 0.703 & $0.41^{*}$ & 11.803 & 0.00 & 0.863 & $0.36^{*}$ & 1.844 & $0.40^{*}$ & 2.424 & 0.10 & 0.050 & 0.908 & 0.41 & 0.019 \\
\hline TMT B Time & 1.292 & $0.27^{*}$ & 0.055 & $0.82^{*}$ & 2.228 & $0.15^{*}$ & 12.752 & 0.00 & 0.535 & 0.52 & 0.004 & 1.564 & 0.23 & 0.012 \\
\hline TMT-B Mistakes & 0.301 & $0.59^{*}$ & 0.299 & $0.59^{*}$ & 0.861 & $0.36^{*}$ & 59.117 & $<0.00$ & 2.504 & 0.13 & 0.035 & 0.206 & 0.66 & 0.003 \\
\hline WAIS-III: Digits & 0.316 & $0.58^{*}$ & 0.021 & $0.58^{*}$ & 0.394 & $0.54^{*}$ & 0.911 & $0.63^{*}$ & 3.632 & $0.04^{*}$ & 0.030 & 3.285 & 0.18 & 0.014 \\
\hline WAIS-III: Digit Symbol & 28.398 & $<0.00$ & 8.209 & 0.01 & 8.241 & 0.01 & 181.195 & $<0.00$ & 1.590 & 0.22 & 0.012 & 1.584 & 0.22 & 0.012 \\
\hline WAIS III: Arithmetic & 0.364 & $0.55^{*}$ & 0.505 & $0.49^{*}$ & 0.304 & $0.59^{*}$ & 2.321 & $0.31^{*}$ & 4.372 & $0.02^{*}$ & 0.038 & 0.380 & 0.69 & 0.003 \\
\hline CAMCOG: Visual Reasoning & 0.003 & $0.96^{*}$ & 0.270 & $0.61^{*}$ & 0.072 & $0.79^{*}$ & 1.833 & $0.40^{*}$ & 0.156 & 0.86 & 0.002 & 0.307 & 0.74 & 0.003 \\
\hline RBMT: Visual Memory & 2.996 & $0.10^{*}$ & 2.155 & $0.16^{*}$ & 1.510 & $0.23^{*}$ & 2.996 & $0.22^{*}$ & 2.308 & 0.11 & 0.018 & 0.002 & 1.0 & $<0.001$ \\
\hline CDT Order & 0.0435 & $0.52 *$ & 0.007 & $0.93^{*}$ & 3.685 & $0.07^{*}$ & 0.601 & $0.74^{*}$ & 0.938 & 0.40 & 0.010 & 1.009 & 0.97 & 0.011 \\
\hline CDT Copy & 4.436 & 0.05 & 5.510 & 0.03 & 7.100 & 0.02 & 0.385 & $0.83^{*}$ & 0.394 & 0.68 & 0.002 & 0.598 & 0.55 & 0.003 \\
\hline SVF & 4.221 & $0.05^{*}$ & 0.394 & $0.54^{*}$ & 3.342 & $0.08^{*}$ & 1.062 & $0.59^{*}$ & 0.655 & 0.53 & 0.005 & 0.920 & 0.41 & 0.007 \\
\hline LVF-P & 0.546 & $0.47^{*}$ & 5.239 & 0.03 & 2.978 & $0.10^{*}$ & 5.544 & $0.06^{*}$ & 1.821 & 0.18 & 0.019 & 0.334 & 0.72 & 0.003 \\
\hline LVF-M & 0.064 & $0.80^{*}$ & 0.193 & $0.67^{*}$ & 0.115 & $0.74^{*}$ & 1.111 & $0.60^{*}$ & 1.572 & 0.22 & 0.010 & 0.535 & 0.59 & 0.003 \\
\hline LVF-R & 0.682 & $0.42^{*}$ & 2.490 & $0.13^{*}$ & 0.263 & $0.61^{*}$ & 5.684 & $0.06^{*}$ & 0.547 & 0.58 & 0.006 & 0.344 & 0.71 & 0.004 \\
\hline GDS & 0.236 & $0.63^{*}$ & 4.104 & $0.06^{*}$ & 1.386 & $0.25^{*}$ & 5.368 & $0.07^{*}$ & 1.697 & 0.20 & 0.020 & 3.414 & $0.04^{*}$ & 0.041 \\
\hline
\end{tabular}

$n=23$. Test of Levene $p \geq 0.05^{*}$; Sphericity $p \geq 0.05^{*}$; ANOVA $p \leq 0.05^{*}$; CAMCOG, Cambridge Cognition Examination; CDT, Clock Drawing Test; LVF, Lexical Verbal Fluency; MMSE, Mini-Mental State Examination; RBMT, Rivermead Behavioural Memory Test; SVF, Semantic Verbal Fluency; TMT, Trail Making Test; WAIS-III, Wechsler Adult Intelligence Scale; GDS, Geriatric Depression Scale.

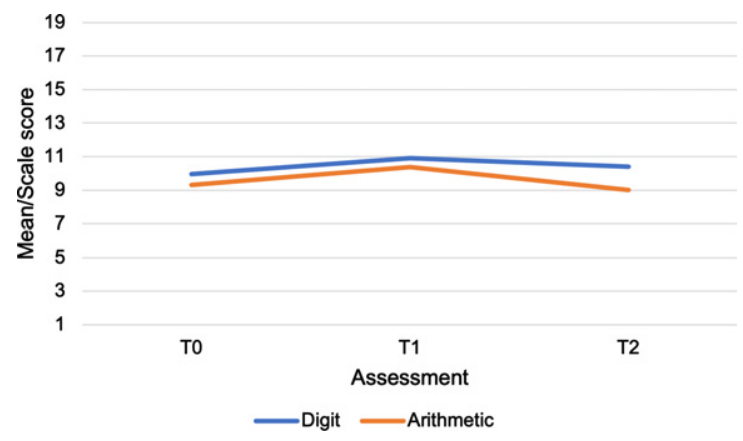

Fig. 2. Analysis with ANOVA. Time factor for Digits and Arithmetic of the Wechsler Adult Intelligence Scale (WAIS-III).

control) in patient performance on the following tests: $\operatorname{MMSE}\left(\mathrm{F}=8.971 ; p=0.03 ; \eta^{2}=0.019\right)$ and the GDS $\left(\mathrm{F}=3.414 ; p=0.04 ; \eta^{2}=0.041\right)$ (Table 4). This analysis showed that the cognitive performance of EG patients was less positively influenced by training than CG patients at the 12-month evaluation in MMSE. While the opposite occurred for the GDS (Fig. 3). Also, small effect sizes were found between time- study group interactions for the following variables: TMT-A-time $\left(\mathrm{F}=1.641 ; p=0.21 ; \eta^{2}=\right.$ $0.021)$ and TMT-A-mistakes $(\mathrm{F}=0.908 ; p=0.41$; $\eta^{2}=0.019$ ) (Table 4, Fig. 3).

Also, we found a significant interaction and a lowmedium effect size between time, study group, and clinical group for the variable CDT Order $(\mathrm{F}=3.455$; $p=0.04 ; \eta^{2}=0.033$ ) (Table 5). This analysis showed that people with MCI in the EG maintained their cognitive performance over a long time and even improved in the case of people with mild dementia, while people with $\mathrm{MCI}$ and mild dementia in the CG tended to decrease their performance with relation to time (Fig. 4).

On the other hand, a low-medium effect size was found for CAMCOG visual reasoning $(\mathrm{F}=2.753$; $\left.p=0.08 ; \eta^{2}=0.030\right)$ and medium for TMT-BMistakes $\left(\mathrm{F}=1.731 ; p=0.20 ; \eta^{2}=0.068\right)$ (Table 5). So, people with MCI and mild dementia of the EG improved their performance in TMT-B-Mistakes over time compared to the CG that decreased in the case of people with MCI or was maintained in the case of people with mild dementia. With respect to Visual Reasoning of CAMCOG, people with EG MCI improved their performance over time compared to CG, contrary to what happened with people with mild dementia (Fig. 4). No adverse effects of GRADIOR were reported in people with MCI and mild dementia.

\section{DISCUSSION}

The purpose of this RCT was to find out the effects of a GRADIOR CCT program on cognitive 


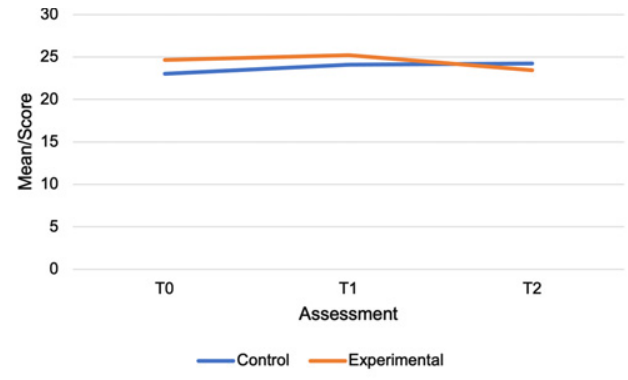

(a)

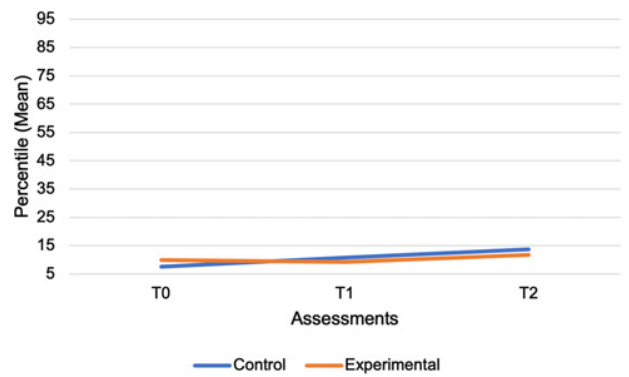

(c)

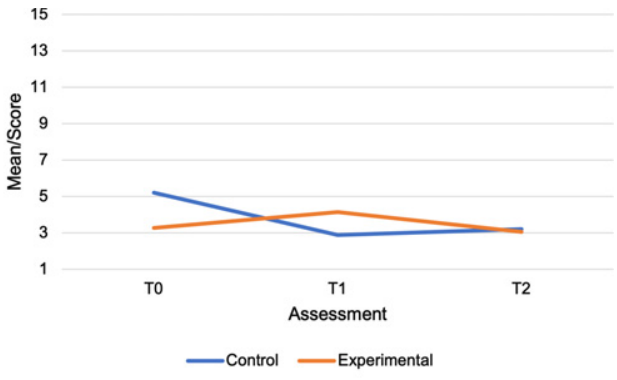

(b)

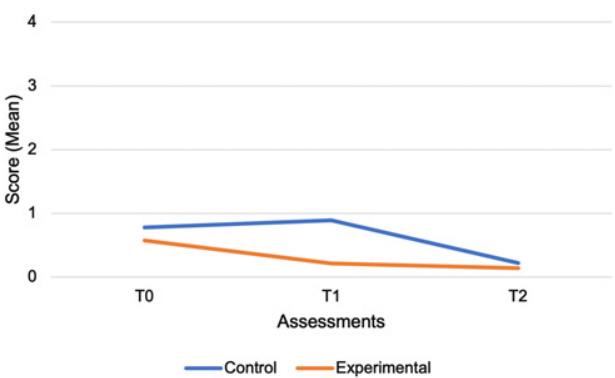

(d)

Fig. 3. Analysis with ANOVA. Time and group study (Experimental and Control) interaction for a) Mini-Mental State Examination (MMSE), b) Geriatric Depression Scale (GDS), c) Trail Making Test (TMT) A Time, and d) TMT A mistakes.

Table 5

Levene's test, sphericity, and ANOVA. Comparison of cognitive performance between $\mathrm{T}_{0}, \mathrm{~T}_{1}-\mathrm{T}_{2}(n=23)$ according to the clinical group (MCI and mild dementia) with respect to the study group (experimental and control)

\begin{tabular}{|c|c|c|c|c|c|c|c|c|c|c|c|}
\hline \multirow[t]{3}{*}{ Test } & \multicolumn{6}{|c|}{ Levene's Test } & \multicolumn{2}{|c|}{ Sphericity } & \multirow{2}{*}{\multicolumn{3}{|c|}{$\begin{array}{c}\text { ANOVA } \\
\mathrm{T}_{0}-\mathrm{T}_{2}{ }^{*} \\
\text { Study Group* } \\
\text { Clinic Group }\end{array}$}} \\
\hline & \multicolumn{2}{|c|}{$\mathrm{T}_{0}$} & \multicolumn{2}{|c|}{$\mathrm{T}_{1}$} & \multicolumn{2}{|c|}{$\mathrm{T}_{2}$} & \multirow[b]{2}{*}{$\chi^{2}$} & \multirow[b]{2}{*}{$p$} & & & \\
\hline & $\mathrm{F}$ & $p$ & $\mathrm{~F}$ & $p$ & $\mathrm{~F}$ & $p$ & & & $\mathrm{~F}$ & $p$ & $\eta^{2}$ \\
\hline MMSE & 1.377 & $0.28^{*}$ & 2.923 & $0.06^{*}$ & 1.533 & $0.24^{*}$ & 3.129 & $0.21^{*}$ & 1.758 & 0.19 & 0.008 \\
\hline ADAS-Cog & 2.202 & $0.12^{*}$ & 1.640 & $0.22^{*}$ & 1.903 & $0.17^{*}$ & 5.798 & $0.06^{*}$ & 0.725 & 0.49 & 0.003 \\
\hline TMT A Time & 1.477 & $0.26^{*}$ & 2.574 & $0.09^{*}$ & 1.981 & $0.16^{*}$ & 17.127 & $<0.001$ & 0.597 & 0.56 & 0.011 \\
\hline TMT-A Mistakes & 3.244 & $0.06^{*}$ & 3.145 & $0.06^{*}$ & 87.750 & $<0.001$ & 2.000 & $0.37^{*}$ & 0.714 & 0.50 & 0.015 \\
\hline TMT B Time & - & - & - & - & - & - & - & - & - & - & - \\
\hline TMT-B Mistakes & 6.304 & 0.01 & 6.299 & 0.01 & 7.231 & 0.01 & 1.292 & $0.52^{*}$ & 1.731 & 0.20 & 0.068 \\
\hline WAIS-III: Digits & 0.026 & $0.99^{*}$ & 1.322 & $0.30^{*}$ & 0.830 & $0.49^{*}$ & 2.430 & $0.30^{*}$ & 1.087 & 0.34 & 0.008 \\
\hline WAIS-III: Digit Symbol & 0.940 & $0.44^{*}$ & 3.362 & 0.04 & 4.378 & 0.02 & 0.406 & $0.81^{*}$ & 1.566 & 0.22 & 0.009 \\
\hline WAIS III: Arithmetic & 1.267 & $0.31^{*}$ & 2.270 & $0.11^{*}$ & 0.602 & $0.62^{*}$ & 1.437 & $0.49^{*}$ & 1.891 & 0.17 & 0.017 \\
\hline CAMCOG: Visual Reasoning & 0.891 & $0.46^{*}$ & 0.909 & $0.45^{*}$ & 0.367 & $0.78^{*}$ & 1.024 & $0.60^{*}$ & 2.753 & 0.08 & 0.030 \\
\hline RBMT: Visual Memory & 1.026 & $0.40^{*}$ & 0.308 & $0.82^{*}$ & 0.911 & $0.45^{*}$ & 3.737 & $0.15^{*}$ & 0.543 & 0.59 & 0.004 \\
\hline CDT Order & 0.163 & $0.92^{*}$ & 4.251 & 0.02 & 0.585 & $0.63^{*}$ & 1.411 & $0.49^{*}$ & 3.455 & $0.04^{*}$ & 0.033 \\
\hline CDT Copy & 2.113 & $0.13^{*}$ & 3.444 & 0.04 & 6.063 & 0.00 & 0.572 & $0.75^{*}$ & 1.175 & 0.32 & 0.006 \\
\hline SVF & 1.612 & $0.22^{*}$ & 0.568 & $0.64^{*}$ & 1.783 & $0.19^{*}$ & 1.188 & $0.55^{*}$ & 0.192 & 0.83 & 0.001 \\
\hline LVF-P & 2.377 & $0.10^{*}$ & 2.589 & $0.09^{*}$ & 2.793 & $0.07^{*}$ & 5.601 & $0.06^{*}$ & 0.958 & 0.39 & 0.010 \\
\hline LVF-M & 1.665 & $0.21^{*}$ & 4.235 & 0.02 & 0.670 & $0.58^{*}$ & 0.316 & $0.85^{*}$ & 0.039 & 0.96 & $<0.001$ \\
\hline LVF-R & 0.675 & $0.58^{*}$ & 2.265 & $0.11^{*}$ & 0.776 & $0.52^{*}$ & 4.855 & $0.09^{*}$ & 1.017 & 0.37 & 0.011 \\
\hline GDS & 0.149 & $0.92^{*}$ & 1.391 & $0.28^{*}$ & 0.887 & $0.47^{*}$ & 5.000 & $0.08^{*}$ & 0.175 & 0.84 & 0.002 \\
\hline
\end{tabular}

$n=23$. Test of Levene $p \geq 0.05^{*}$; Sphericity $p \geq 0.005^{*}$; ANOVA $p \leq 0.05^{*}$; CAMCOG, Cambridge Cognition Examination; CDT, Clock Drawing Test; LVF, Lexical Verbal Fluency; MMSE, Mini-Mental State Examination; RBMT, Rivermead Behavioural Memory Test; SVF, Semantic Verbal Fluency; TMT, Trail Making Test; WAIS-III, Wechsler Adult Intelligence Scale; GDS, Geriatric Depression Scale. 


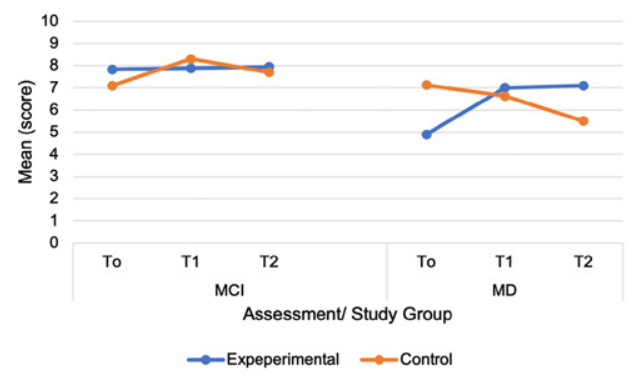

(a)

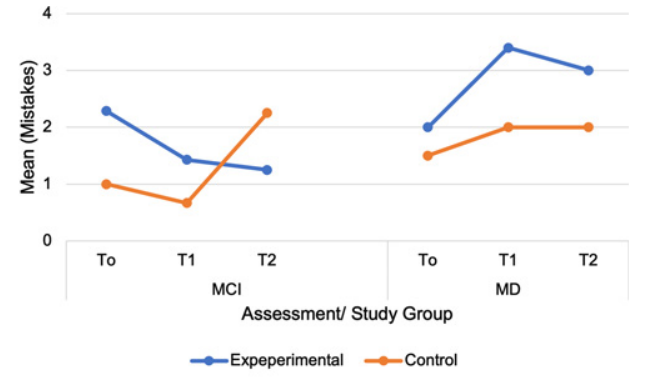

(b)

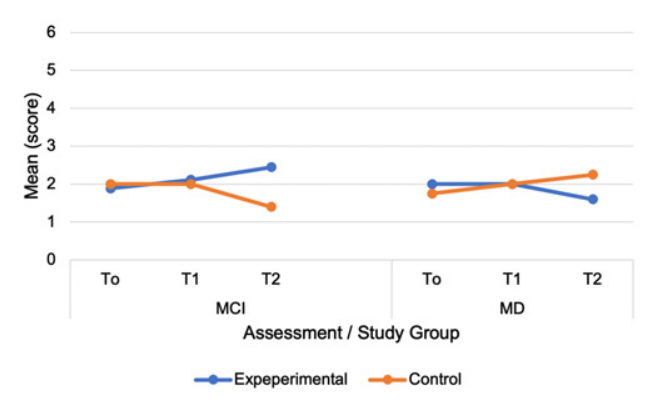

(c)

Fig. 4. Analysis with ANOVA. Time, Clinical Group (MCI and Mild dementia), and Study Group (Experimental and Control) interaction for a) Clock Drawing Test (CDT) Order, b) Trail Making Test (TMT) B mistakes, and c) Visual Reasoning of Cambridge Cognition Examination (CAMCOG).

and emotional aspects in people with MCI or mild dementia. Regarding the general cognitive performance obtained from the MMSE, an instrument used for the selection and during the follow-up evaluations in the sample, we observe an improvement trend at 4 months with respect to the baseline and a worsening trend at 12 months with respect to 4 months in the EG. Although this seems to underestimate the effect of CCT at first, it was important for us to evaluate cognitive functions and processes by using different specific tests for this objective.

One of the cognitive functions most evaluated was EF. In this order of ideas, both groups exhibited alterations in visual reasoning (CAMCOG) at the beginning of the study, which could be attributed to the sample's low educational level. However, the EG had a better performance than the CG in visual reasoning (CAMCOG) at 4 months (intergroup). Also, we saw longitudinal changes in cognitive performance within each group (intragroup). The CG showed a deterioration trend towards 12 months compared to 4 months, while the EG slightly improved at 4-12 months of CCT. Likewise, with respect to the EG, we found that people with MCI improved their performance while people with mild dementia worsened over time compared to the CG.
This could be explained because the GRADIOR cognitive training plan included activities such as puzzles; possibly this allowed training this EF. This means that the treatment contributed to a slight improvement in this process, preventing an even greater deterioration in the EG.

Our results suggesting an improvement trend in selective-sustained attention (evaluated by TMT-Amistakes) at 4 months with respect to baseline and thereafter in processing speed (evaluated by TMTA-time) at 12 months with respect to 4 months in people attending the GRADIOR sessions. Also, the results showed an interaction between "time and study group"; in this way, the EG slightly worsens its performance in TMT-A-time at 4 months, but makes fewer errors at 12 months, compared to CG, where the opposite happens. This means that these people found "doing it well" more important than "doing it fast" and therefore prioritized and intensified their attention capacity while performing the task. The improvement in attentional processing has also been noted in other studies such as that by Hagovská et al. [17] after 10 weeks of CCT and González-Palau et al. [16] after 12 weeks of combined (cognitive and physical) training delivered to healthy older adults and older adults with MCI. 
The improvement in processing speed has been explained as the result of stimulation associated with social interaction in people with MCI [20]. According to our study, the improvement in processing speed after 12 months with respect to 4 months in the EG could have been due to the increase in social interaction during the CCT. The way in which GRADIOR was applied, in a shared room where users worked with their customized programs [50], probably favored the increase in social interaction among the people with MCI and mild dementia that attended the CCT $[44,53]$. Further research is required to define the relationship between processing speed and social interaction.

The EG showed an improvement in selectivedivided attention, alternation, cognitive flexibility, inhibitory control, and cognitive flexibility (evaluating processes by the TMT-B-mistakes) after 12 months of CCT with respect to 4 months (intragroup). Likewise, the results highlighted that the group of people with MCI and mild dementia associated with the EG improved in these processes over time compared to the CG. Studies also mentioned an enhancement in these processes in people with MCI [20]. This could be explained by the fact that GRADIOR is a CCT program that includes one of the basic training principles: "feedback" [54]. GRADIOR delivers feedback on the individual's performance in each of the activities, which offers the person the chance to "learn" and correct the mistake immediately and/or soon after making it [50].

In the field of neuropsychology, mistakes of omission or commission are a relevant source of qualitative information in the assessment of cognitive processes. People with MCI or mild dementia are often aware of these mistakes and, therefore, probably of their deficits. The purpose of any treatment, CCT or therapeutic process is that the patient, depending on the level of decline, be aware of his/her deficit. If otherwise, we would be dealing with anosognosia $[55,56]$.

As compared to the TMT-A, the EG took longer in completing the TMT-B at 4 months, which suggests low processing speed, although this decreases with age $[57,58]$. The EG probably made more mental checks to avoid mistakes and were more aware of their difficulties, something that could be observed with greater precision during the evaluation at 12 months, which contributed to these results, considering the complex nature of the task of the B-condition of the TMT.
The overall improvement in EF after using a CCT program was also noted by Shatil et al. [59], although these authors only included healthy older adults in their study. In our study, there were also changes associated with EF such as fluency, categorization, and monitoring of performance evaluated by SVF and LVF, although these were observed at two different stages of the intervention. While the EG showed an improvement trend at 4 months in SVF tasks, at 12 months, this same group improved in LVF-P tasks. This was not so in the CG. This is interesting, the form of gradual improvement in verbal fluency, semantic fluency being the first to show improvement and then phonological fluency. The latter being in most cases the most compromised in PwD [60].

Calculation and numerical reasoning (WAIS-III Arithmetic) were one of the best-preserved functions in people with MCI and mild dementia at baseline. Both groups showed an improvement trend at 4 months and a slight worsening at 12 months. In this regard, the improvement was greater in the EG and the worsening in the CG. It is worth noting that one of the reasons why calculation is one of the best-preserved abilities among this population group could be related to their living conditions and, especially, to the need of developing and training this ability due to its multiple uses in various areas of daily living, from activities like shopping to more professional uses. Therefore, activities of daily living contribute to preserving the most frequently used functions.

One of the commonly deteriorated functions in people with $\mathrm{MCI}$ and mild dementia is memory [57, 61]. We only managed to perceive an improvement trend at 12 months with respect to 4 months in visual recognition tasks (RMBT) in both groups. Nevertheless, the EG obtained better results than the CG at 12 months. This is in line with the study conducted by Hwang et al. [62], where a significant increase in visual recognition was reported in people with Alzheimer's disease. It is a simple cognitive task that seems to benefit from any psychosocial intervention, both the usual and the more specific using GRADIOR. Moreover, considering that people with MCI accounted for a large percentage of our sample, recognition tasks were often better retained than free-recall ones [63].

Different studies have also reported significant alterations in the working memory (WM) of people with MCI and mild dementia [64, 65]. In our study, WM at 4 months appeared well-preserved in both groups, although the EG improved at 12 months in 
Digit-Symbol test of WAIS-III compared to 4 months (intragroup). CG worsened at 12 months with respect to 4 months in two tests (Digit and Digit-Symbol of WAIS-III) (intragroup).

According to Hyer et al. [18], the WM in people with MCI improved after 7 weeks of CCT. Studies based on shorter periods of CCT and focused on WM only report improvements in this function in people with MCI $[19,24]$. Other studies noted changes in the two variants of the WAIS-III Digits Test. Cavallo et al. [14] found that performance was better in forward and backwards spans of the Digits test at 12 weeks of CCT and that it was maintained at 6 months in people with Alzheimer's disease. This last finding was not explicit in our study, where the overall score on the WAIS-III Digits Test was considered.

Regarding Visuoconstructive ability of CDT, both groups performed similarly at baseline and at 4 months. However, the CG showed a worsening trend at 12 months with respect to 4 months, while the EG maintained similar levels of performance at 4-12 months (although the latter was not significant). However, a significant interaction was found which made it relevant that the EG of people with MCI maintained their performance and the group of people with mild dementia slightly improved at 4 months and maintaining over time compared to people with MCI and mild dementia of CG, this group decreased their performance.

Finally, the EG experienced an improvement in mood at 12 months of CCT with respect to 4 months. Also, there was a significance interaction between the CG-EG with a small effect size, where the EG scored better than the CG on the GDS at 12 months. Our findings are even related to meta-analyses such as that by García-Casal et al. [21] who also mentioned a small effect size on the improvement of depression in PwD after a CCT program. Or also, studies that reported an improvement in mood in people with MCI [16], even if others consider that this improvement is not possible [66].

\section{Recommendations and limitations}

The results reveal that the functioning and improvement of both groups at 4 months was similar, these data being inconclusive as to the short-term benefits of using this CCT program. Only after 12 months of CCT did the results show slight improvement and/or maintenance trends in certain cognitive processes and mood, with moderate-large effect sizes and, in certain cases, statistical significance in EG. In contrast, the CG showed greater worsening tendencies towards 12 months.

The above might be explained based on how the CCT GRADIOR was implemented: the first 3 months of CCT consisted of a standard plan, after which treatment was tailored to each individual's cognitive level over the 12 months. Such customization could have provided more benefits and effectiveness insofar as it was adjusted to the cognitive needs and characteristics of each participant. GRADIOR suggests and recommends a series of criteria to serve as the basis to make changes in the difficulty level of each task [50].

Flak et al. [67] suggested a comparison between customized and nonadaptive training and their influence on the WM of patients with MCI. Nevertheless, they found no changes after 5 weeks of CCT. By contrast, the study by Peretz et al. [68] proved the effectiveness of a customized CCT plan at 3 months on improving visuospatial WM, visuospatial learning, and sustained attention in older adults. While these studies provide an interesting approach, it would be advisable to consider in any cognitive training, the design of a training plan personalized to the individual's cognitive profile and characteristics.

Another relevant aspect was that the sample consisted of people with MCI, which is sometimes spontaneously reversible, and mild dementia, whose natural progress is not towards rapid decline. The benefits of applying a specific program CCT such as GRADIOR in cases of MCI and mild dementia will be more relevant when applied over long periods of time [24], against what is commonly the case with the implementation of these programs in studies based on few weeks of intervention [26, 69].

Lee et al. [25] recommended studies with longer periods of CCT be undertaken. Nevertheless, it is necessary to be aware of the difficulties involved in completing RCTs with older adults and long CCT programs. Such studies are often challenged by different factors that may be biological (high rates of mortality), physical (mobility alterations), contextual (little understanding of the family about the disease and its treatment), psychological (emotional disturbances that hinder treatment adherence), social (stigma associated with the person's social network), environmental (pandemic outbreak), and economic (lack of funding, high costs of human resources and infrastructures).

The relevance of this RCT is its 12-month length, so that, in addition to customizing the CCT, the purpose was continuity in time with frequent sessions 
over an extended period to test whether there were significant changes in cognitive processes and/or if they were maintained over time. If so, the use of a continuous CCT program would be related to the progressive nature of the neurodegenerative process that occurs in dementia, although this process varies among individuals and CCT cannot guarantee full recovery of cognitive processes.

One of the study's limitations is that the sample was small. Although the initial sample was 89 people, only 62 individuals managed to complete the 4 months and our attempt to have the sample complete the 12 months was hampered by the consequences of the outbreak and the extension in time of the COVID19 pandemic.

The pandemic led to the closure of many public health centers and, therefore, the people undergoing the CCT with GRADIOR stopped attending the sessions. Only 23 individuals managed to complete the 12-months scheduled for the CCT before the pandemic began and therefore, the CG people on the waiting list were unable to start the treatment. This situation helps to redefine its current implementation and strengthens lines of research that are already in progress to consolidate the use of portable devices at home to make these programs more accessible to older adults [70], developing CCT programs based on a user-center methodology [71], and implementing digital literacy programs to bridge the digital divide [72]. Shatil et al. [59] suggested TV-based computer literacy training for older adults. Nevertheless, it will be necessary to develop devices.

We are aware that CCT is not the only treatment option for people with MCI and mild dementia, since the literature includes studies assessing the effectiveness of these cognitive programs combined with physical training programs [73] or other approaches such as reminiscence therapy [74] on different alterations associated with the disease. Dementia is a neurodegenerative disease that causes changes at different functional levels [75], so it would be interesting to engage in future studies to assess the effectiveness of GRADIOR in combination with other types of program [44]. In short, we believe that a multidisciplinary approach could prove more beneficial for this population [10].

GRADIOR can be considered a promising CCT program, it is a 1) flexible program that has not only been developed to be used with people with dementia, but also with people with other neurological and/or psychiatric pathologies; 2) it is easy to use for people with little knowledge in technology; 3) useful, due to its effectiveness, degree of usability [47] and level of user experience [48]. Specifically, it allows the 4) construction of a neuropsychological profile of each user from a neuropsychological evaluation that can be carried out from the same program; 5) design and implementation of a cognitive stimulation plan based on altered processes and the user's cognitive level; 6) adjustment of the treatment plan according to the user's cognitive performance.

Also, and considering our results, the use of GRADIOR is recommended in people with MCI and mild dementia for long periods of time. Cognitive training should be continuous and maintained over time due to the characteristics of the cognitive deterioration that these people present. We consider that its interruption could have a negative impact on the cognitive state of the person.

\section{Implications}

This study has important methodological, clinical, and practical implications in the field of neuropsychology, new technologies, and dementias. Methodological implications in terms of trying to imply a representative sample size and with it the multiple factors that could make sample collection difficult, which could be taken into consideration by future studies. Also, the application and implementation of a "GRADIOR" CCT program for long periods of time (12 months) and its positive influence on the maintenance of cognitive processes. Both the sample size and the continuity of treatment are two factors that any clinical trial whose objective is to evaluate the effectiveness should try to incorporate. These are also factors that have constituted part of the limitations of most of the studies currently published on the subject.

Perhaps the scope of this RCT could be questioned due to the changes in the design mentioned in the methodology session and the final size of the sample, but we consider that this RCT maintained its methodological rigor and presented an effort to want to overcome the limitation associated with the short training time proposed in most RCTs. We know that maintaining an RCT of 12 months requires a cost of great magnitude with respect to human, technological, and infrastructure resources. But above all, maintaining the level of sample motivation for them to attend the CCT sessions requires, for example, that the CCT be interesting and personalized to the needs of the people. And although we reported a sample loss around 12 months, this was not due to the nature 
of the RCT, but to the impossibility of continuing due to external factors, specifically due to COVID- 19 .

Regarding the clinical implications, cognitive rehabilitation has proven to be a field that responds to certain needs of people with dementia from the use of pencil and paper to the design and development of CCT programs such as GRADIOR that contribute to training, maintenance, and improvement of cognitive performance and delay of progressive deterioration in people with dementia. GRADIOR is a program that is adapted to the type and level of cognitive impairment, aspects that contribute not only to its effectiveness, but also to its usability. The use of tools such as GRADIOR makes the work of the therapist easier, who could have a session with several patients at the same time.

Likewise, this study indicates a tool, whose practical implication corresponds to its degree of accessibility that prevents these people from starting a cognitive training plan. Although this point is currently being improved, to increase its degree of accessibility. Likewise, this study contributes to future studies on effectiveness with respect to methodological aspects.

\section{Conclusion}

Some intergroup differences in cognitive performance were detected with respect to each of the evaluation times, visual reasoning (CAMCOG) being one of them. Likewise, improvement trends were identified at the intragroup level between baseline, 4 months, and 12 months for EG with respect to most of the cognitive processes evaluated by the different tests and although something similar happened for CG at 4 months, this same group showed changes and a tendency to worsen towards 12 months in most of the tests. We also highlight the interaction between "time and group", showing GRADIOR a positive impact on mood (GDS) and sustained attention (TMT-A-mistakes) in EG. And a significant interaction between "time, study group, and clinic group" for the CDT Order, highlighting the positive impact of the GRADIOR sessions in people with MCI and mild dementia of EG.

The effects seem to be more significant in those cases where the 12-month treatment was completed, which is why it is advisable to use this type of programs for long periods to test their effects. Many patients were unable to complete the scheduled sessions because of the COVID-19 pandemic, which also highlights the relevance of implementing these intervention programs through more accessible technologies.

\section{ACKNOWLEDGMENTS}

The research presented in this paper was carried out as part of the Marie Curie Initial Training Network (ITN) action, H2020-MSCA-ITN-2015, under grant agreement number 676265. INDUCT: Interdisciplinary Network for Dementia Using Current Technology. H2020 Marie Skłodowska Curie Actions - Innovative Training Networks, 2015 [1]. And, under grant agreement number 813196. DISTINCT: Dementia: Intersectorial Strategy for Training and Innovation Network for Current Technology [2]. Department of Research and Development, INTRAS Foundation, Zamora, Spain.

Authors' disclosures available online (https:// www.j-alz.com/manuscript-disclosures/21-5350r1).

\section{SUPPLEMENTARY MATERIAL}

The supplementary material is available in the electronic version of this article: https://dx.doi.org/ 10.3233/JAD-215350.

\section{REFERENCES}

[1] Weintraub S, Wicklund AH, Salmon DP (2012) The neuropsychological profile of Alzheimer disease. Cold Spring Harb Perspect Med 2, a006171.

[2] Pedroso RV, Corazza DI, Andreatto CAA, da Silva TMV, Costa JLR, Santos-Galduróz RF (2018) Cognitive, functional and physical activity impairment in elderly with Alzheimer's disease. Dement Neuropsychol 12, 28-34.

[3] Kitching D (2015) Depression in dementia. Aust Prescr 38, 209-211.

[4] Wang L, Larson EB, Bowen JD, van Belle G (2006) Performance-based physical function and future dementia in older people. Arch Intern Med 166, 1115-1120.

[5] Hackett RA, Steptoe A, Cadar D, Fancourt D (2019) Social engagement before and after dementia diagnosis in the English Longitudinal Study of Ageing. PLoS One 14, e0220195.

[6] Campbell NL, Unverzagt F, LaMantia MA, Khan BA, Boustani MA (2013) Risk factors for the progression of mild cognitive impairment to dementia. Clin Geriatr Med 29, 873-893.

[7] Pal K, Mukadam N, Petersen I, Cooper C (2018) Mild cognitive impairment and progression to dementia in people with diabetes, prediabetes and metabolic syndrome: A systematic review and meta-analysis. Soc Psychiatry Psychiatr Epidemiol 53, 1149-1160.

[8] Roberts RO, Knopman DS, Mielke MM, Cha RH, Pankratz VS, Christianson TJ, Geda YE, Boeve BF, Ivnik RJ, Tangalos EG, Rocca WA, Petersen RC (2014) Higher risk of progression to dementia in mild cognitive impairment cases who revert to normal. Neurology 82, 317-325. 
[9] Huang LK, Chao SP, Hu CJ (2020) Clinical trials of new drugs for Alzheimer disease. J Biomed Sci 27, 18.

[10] Vernooij-Dassen M, Moniz-Cook E, Verhey F, Chattat R, Woods B, Meiland F, Franco M, Holmerova I, Orrell M, de Vugt M (2021) Bridging the divide between biomedical and psychosocial approaches in dementia research: The 2019 INTERDEM manifesto. Aging Ment Health 25, 206-212.

[11] Meiland F, Innes A, Mountain G, Robinson L, van der Roest H, García-Casal JA, Gove D, Thyrian JR, Evans S, Dröes RM, Kelly F, Kurz A, Casey D, Szcześniak D, Dening T, Craven MP, Span M, Felzmann H, Tsolaki M, Franco-Martin M (2017) Technologies to support community-dwelling persons with dementia: A position paper on issues regarding development, usability, effectiveness and cost-effectiveness, deployment, and ethics. JMIR Rehabil Assist Technol 4, e1.

[12] Schaller S, Marinova-Schmidt V, Gobin J, Criegee-Rieck M, Griebel L, Engel S, Stein V, Graessel E, KolominskyRabas PL (2015) Tailored e-Health services for the dementia care setting: A pilot study of 'eHealthMonitor'. BMC Med Inform Decis Mak 15, 58.

[13] Yousaf K, Mehmood Z, Saba T, Rehman A, Munshi AM, Alharbey R, Rashid M (2019) Mobile-health applications for the efficient delivery of health care facility to People with Dementia (PwD) and Support to their carers: A survey. Biomed Res Int 2019, 7151475.

[14] Cavallo M, Hunter EM, van der Hiele K, Angilletta C (2016) Computerized structured cognitive training in patients affected by early-stage Alzheimer's disease is feasible and effective: A randomized controlled study. Arch Clin Neuropsychol 31, 868-876.

[15] Gaitán A, Garolera M, Cerulla N, Chico G, RodriguezQuerol M, Canela-Soler J (2013) Efficacy of an adjunctive computer-based cognitive training program in amnestic mild cognitive impairment and Alzheimer's disease: A single-blind, randomized clinical trial. Int J Geriatr Psychiatry 28, 91-99.

[16] González-Palau F, Franco M, Bamidis P, Losada R, Parra E, Papageorgiou SG, Vivas AB (2014) The effects of a computer-based cognitive and physical training program in a healthy and mildly cognitive impaired aging sample. Aging Ment Health 18, 838-846.

[17] Hagovská M, Dzvoník O, Olekszyová Z (2017) Comparison of two cognitive training programs with effects on functional activities and quality of life. Res Gerontol Nurs 10, 172-180.

[18] Hyer L, Scott C, Atkinson MM, Mullen CM, Lee A, Johnson A, McKenzie LC (2016) Cognitive training program to improve working memory in older adults with MCI. Clin Gerontol 39, 410-427.

[19] Vermeij A, Claassen JA, Dautzenberg PL, Kessels RP (2016) Transfer and maintenance effects of online workingmemory training in normal ageing and mild cognitive impairment. Neuropsychol Rehabil 26, 783-809.

[20] Djabelkhir L, Wu YH, Vidal JS, Cristancho-Lacroix V, Marlats F, Lenoir H, Carno A, Rigaud AS (2017) Computerized cognitive stimulation and engagement programs in older adults with mild cognitive impairment: Comparing feasibility, acceptability, and cognitive and psychosocial effects. Clin Interv Aging 12, 1967-1975.

[21] García-Casal JA, Loizeau A, Csipke E, Franco-Martín M, Perea-Bartolomé MV, Orrell M (2017) Computer-based cognitive interventions for people living with dementia: A systematic literature review and meta-analysis. Aging Ment Health 21, 454-467.
[22] Irazoki E, Contreras-Somoza LM, Toribio-Guzmán JM, Jenaro-Río C, van der Roest H, Franco-Martín MA (2020) Technologies for cognitive training and cognitive rehabilitation for people with mild cognitive impairment and dementia. A systematic review. Front Psychol 11, 648 .

[23] Klimova B, Maresova P (2017) Computer-based training programs for older people with mild cognitive impairment and/or dementia. Front Hum Neurosci 11, 262.

[24] Yang HL, Chu H, Kao CC, Chiu HL, Tseng IJ, Tseng P, Chou KR (2019) Development and effectivenes of virtual interactive working memory training for older people with mild cognitive impairment: A single-blind randomised controlled trial. Age Ageing 48, 519-525.

[25] Lee GJ, Bang HJ, Lee KM, Kong HH, Seo HS, Oh M, Bang M (2018) A comparison of the effects between 2 computerized cognitive training programs, Bettercog and COMCOG, on elderly patients with MCI and mild dementia: A singleblind randomized controlled study. Medicine (Baltimore) 97, e13007.

[26] Gates NJ, Rutjes AWS, Di Nisio M, Karim S, Chong LY, March E, Martínez G, Vernooij RWM (2020) Computerised cognitive training for 12 or more weeks for maintaining cognitive function in cognitively healthy people in late life. Cochrane Database Syst Rev 2, CD012277.

[27] Vanova M, Irazoki E, García-Casal JA, Martínez-Abad F, Botella C, Shiells KR, Franco-Martín MA (2018) The effectiveness of ICT-based neurocognitive and psychosocial rehabilitation programmes in people with mild dementia and mild cognitive impairment using GRADIOR and ehcoBUTLER: Study protocol for a randomised controlled trial. Trials 19, 100 .

[28] Franco-Martín MA (2017) Evaluation of GRADIOR, a neuropsychological rehabilitation programme for people with mild dementia and mild cognitive impairment. ISRCTN Registry

[29] Petersen RC (2011) Clinical practice. Mild cognitive impairment. N Engl J Med 364, 2227-2234.

[30] American Psychiatric Association (2013) Diagnostic and statistical manual of mental disorders (5th ed.), Arlington, VA.

[31] Blesa R, Pujol M, Aguilar M, Santacruz P, Bertran-Serra I, Hernandez G, Sol JM, Pena-Casanova J; NORMACODEM Group. NORMAlisation of Cognitive and Functional Instruments for DEMentia (2001) Clinical validity of the 'mini-mental state' for Spanish speaking communities. Neuropsychologia 39, 1150-1157.

[32] Folstein MF, Folstein SE, McHugh PR (1975) "Mini-mental state": A practical method for grading the cognitive state of patients for the clinician. J Psychiatr Res 12, 189-198.

[33] Rosen WG, Mohs RC, Davis KL (1984) A new rating scale for Alzheimer's disease. Am J Psychiatry 141, 1356-1364.

[34] Tombaugh TN (2004) Trail making test A and B: Normative data stratified by age and education. Arch Clin Neuropsychol 19, 203-214.

[35] Kokmen E, Smith GE, Petersen RC, Tangalos E, Ivnik RC (1991) The short test of mental status. Correlations with standardized psychometric testing. Arch Neurol 48, 725728.

[36] Wechsler D (1997) WAIS-III administration and scoring manual. The Psychological Corporation

[37] Wilson B, Cockburn J, Baddeley A, Hiorns R (1989) The development and validation of a test battery for detecting and monitoring everyday memory problems. J Clin Exp Neuropsychol 11, 855-870. 
[38] Roth M, Tym E, Mountjoy CQ, Huppert FA, Hendrie H, Verma S, Goddard R (1986) CAMDEX. A standardised instrument for the diagnosis of mental disorder in the elderly with special reference to the early detection of dementia. $\mathrm{Br}$ J Psychiatry 149, 698-709.

[39] Benton AL (1968) Differential behavioral effects in frontal lobe disease. Neurosychologia 6, 53-60.

[40] Peña-Casanova J, Quiñones-Ubeda S, Gramunt-Fombuena N, Quintana-Aparicio M, Aguilar M, Badenes D, Cerulla N, Molinuevo JL, Ruiz E, Robles A, Barquero MS, Antúnez C, Martínez-Parra C, Frank-García A, Fernández M, Alfonso V, Sol JM, Blesa R; NEURONORMA Study Team (2009) Spanish Multicenter Normative Studies (NEURONORMA Project): Norms for verbal fluency tests. Arch Clin Neuropsychol 24, 395-411.

[41] Sheikh JI, Yesavage JA (1986) Geriatric Depression Scale (GDS): Recent evidence and development of a shorter version. Clin Gerontol 5, 165-173.

[42] Franco-Martín M, Palau FG, Ruiz Y, Vargas E, Solis A, Mellado JG, Bartolomé L (2011) Usability of a cognitive (gradior) and physical training program based in new software technologies in patients with mild dementia, mild cognitive impairment and healthy elderly people: Long lasting memories preliminary findings. Neurosci Lett 500(Suppl), e6.

[43] Fumero Vargas G, Franco Martín MA, Perea Bartolomé MV (2009) Puesta en marcha y estudio de usabilidad de un programa de rehabilitación cognitiva por ordenador "GRADIOR" en el tratamiento de déficits neurocognitivo. Departamento de Psicología básica, psicobiologia y metodología de las ciencias del comportamiento, Universidad de Salamanca.

[44] Góngora Alonso S, Toribio Guzmán JM, Sainz de Abajo B, Muñoz Sánchez JL, Martín MF, de la Torre Díez I (2019) Usability evaluation of the eHealth Long Lasting Memories program in Spanish elderly people. Health Informatics $J \mathbf{2 6}$, 1728-1741.

[45] Toribio-Guzmán JM, Parra Vidales E, Viñas Rodríguez MJ, Bueno Aguado Y, Cid Bartolomé MT, Franco-Martín MA (2018) Rehabilitación cognitiva por ordenador en personas mayores: Programa Gradior. Edic Univ Salamanca 24, 61-75.

[46] González-Palau F, Franco M, Toribio-Guzmán JM, Losada R, Parra Vidales E, Bamidis P (2013) Designing a computer-based rehabilitation solution for older adults: The importance of testing usability. PsychNol J 11, 119-136.

[47] Góngora Alonso S, Fumero Vargas G, Morón Nozaleda L, Sainz de Abajo B, de la Torre Díez I, Franco M (2020) Usability analysis of a system for cognitive rehabilitation, "Gradior", in a Spanish region. Telemed J E Health 26, 671-682.

[48] Irazoki E, Sánchez-Gómez MC, Contreras-Somoza LM, Toribio-Guzmán JM, Martín-Cilleros MV, Verdugo-Castro S, Jenaro-Río C, Franco-Martín MA (2021) A qualitative study of the cognitive rehabilitation program GRADIOR for people with cognitive impairment: Outcomes of the focus group methodology. J Clin Med 10, 859.

[49] Toribio Guzmán JM (2016) Long Lasting Memories, una plataforma TIC integrada contra el deterioro cognitivo relacionado con la edad: Estudio de usabilidad (Thesis). Universidad de Salamanca.

[50] Franco-Martin MA, Diaz-Baquero AA, Bueno-Aguado Y, Cid-Bartolomé MT, Parra Vidales E, Perea Bartolomé MV, de la Torre Díez I, van der Roest HG (2020) Computerbased cognitive rehabilitation program GRADIOR for mild dementia and mild cognitive impairment. New features. BMC Med Inform Decis Mak 20, 274.

[51] IBM-Corp (2017) IBM SPSS Statistics for Windows. IBM Corp, NY.

[52] Geisser S, Greenhouse SW (1958) An extension of Boxís results on the use of the $\mathrm{F}$ distribution in multivariate analysis. Ann Math Stat 29, 885-891.

[53] Pinto-Bruno ÁC, García-Casal JA, Csipke E, Jenaro-Río C, Franco-Martín M (2017) ICT-based applications to improve social health and social participation in older adults with dementia. A systematic literature review. Aging Ment Health 21, 58-65.

[54] Adcock M, Sonder F, Schättin A, Gennaro F, de Bruin ED (2020) A usability study of a multicomponent video gamebased training for older adults. Eur Rev Aging Phys Act 17,3 .

[55] Castrillo Sanz A, Andrés Calvo M, Repiso Gento I, Izquierdo Delgado E, Gutierrez Ríos R, Rodríguez Herrero R, Rodríguez Sanz F, Tola-Arribas MA (2016) Anosognosia in Alzheimer disease: Prevalence, associated factors, and influence on disease progression. Neurologia 31, 296-304.

[56] Tagai K, Nagata T, Shinagawa S, Shigeta M (2020) Anosognosia in patients with Alzheimer's disease: Current perspectives. Psychogeriatrics 20, 345-352.

[57] Andrés P, Vico H, Yáñez A, Siquier A, Ferrer GA (2019) Quantifying memory deficits in amnestic mild cognitive impairment. Alzheimers Dement (Amst) 11, 108-114.

[58] Eckert MA, Keren NI, Roberts DR, Calhoun VD, Harris KC (2010) Age-related changes in processing speed: Unique contributions of cerebellar and prefrontal cortex. Front Hum Neurosci 4, 10.

[59] Shatil E, Mikulecká J, Bellotti F, Bureš V (2014) Novel television-based cognitive training improves working memory and executive function. PLoS One 9, e101472.

[60] Laisney M, Matuszewski V, Mézenge F, Belliard S, de la Sayette V, Eustache F, Desgranges B (2009) The underlying mechanisms of verbal fluency deficit in frontotemporal dementia and semantic dementia. J Neurol 256, 1083-1094.

[61] Hildebrandt H, Fink F, Kastrup A, Haupts M, Eling P (2013) Cognitive profiles of patients with mild cognitive impairment or dementia in Alzheimer's or Parkinson's disease. Dement Geriatr Cogn Dis Extra 3, 102-112.

[62] Hwang JH, Cha HG, Cho YS, Kim TS, Cho HS (2015) The effects of computer-assisted cognitive rehabilitation on Alzheimer's dementia patients memories. J Phys Ther Sci 27, 2921-2923.

[63] De Simone MS, Perri R, Fadda L, Caltagirone C, Carlesimo GA (2019) Predicting progression to Alzheimer's disease in subjects with amnestic mild cognitive impairment using performance on recall and recognition tests. J Neurol 266, 102-111.

[64] Kessels R, Overbeek A, Bouman Z (2015) Assessment of verbal and visuospatial working memory in mild cognitive impairment and Alzheimer's dementia. Dement Neuropsychol 9, 301-305.

[65] Kirova AM, Bays RB, Lagalwar S (2015) Working memory and executive function decline across normal aging, mild cognitive impairment, and Alzheimer's disease. Biomed Res Int 2015, 748212 .

[66] Finn M, McDonald S (2012) Computerised cognitive training for older persons with mild cognitive impairment: A pilot study using a randomised controlled trial design. Brain Impair 12, 187-199.

[67] Flak MM, Hol HR, Hernes SS, Chang L, Engvig A, Bjuland KJ, Pripp A, Madsen BO, Knapskog AB, Ulstein I, Lona T, 
Skranes J, Løhaugen GCC (2019) Adaptive computerized working memory training in patients with mild cognitive impairment. A randomized double-blind active controlled trial. Front Psychol 10, 807.

[68] Peretz C, Korczyn AD, Shatil E, Aharonson V, Birnboim S, Giladi N (2011) Computer-based, personalized cognitive training versus classical computer games: A randomized double-blind prospective trial of cognitive stimulation. $\mathrm{Neu}$ roepidemiology 36, 91-99.

[69] Bahar-Fuchs A, Webb S, Bartsch L, Clare L, Rebok G, Cherbuin N, Anstey KJ (2017) Tailored and adaptive computerized cognitive training in older adults at risk for dementia: A randomized controlled trial. J Alzheimers Dis 60, 889-911.

[70] Blackwood J, Shubert T, Fogarty K, Chase C (2016) The impact of a home-based computerized cognitive training intervention on fall risk measure performance in community dwelling older adults, a pilot study. J Nutr Health Aging $\mathbf{2 0}$, 138-145.

[71] Diaz Baquero AA, Dröes R-M, Perea Bartolomé MV, Irazoki E, Toribio-Guzmán JM, Franco-Martín MA, van der Roest H (2021) Methodological designs applied in the development of computer-based training programs for the cognitive rehabilitation in people with Mild Cognitive Impairment (MCI) and mild dementia. Systematic review. J Clin Med 10, 1222.
[72] Martins Van Jaarsveld G (2020) The effects of COVID-19 among the elderly population: A case for closing the digital divide. Front Psychiatry 11, 577427.

[73] Hagovská M, Olekszyová Z (2016) Impact of the combination of cognitive and balance training on gait, fear and risk of falling and quality of life in seniors with mild cognitive impairment. Geriatr Gerontol Int 16, 1043-1050.

[74] Barban F, Annicchiarico R, Pantelopoulos S, Federici A, Perri R, Fadda L, Carlesimo GA, Ricci C, Giuli S, Scalici F, Turchetta CS, Adriano F, Lombardi MG, Zaccarelli C, Cirillo G, Passuti S, Mattarelli P, Lymperopoulou O, Sakka P, Ntanasi E, Moliner R, Garcia-Palacios A, Caltagirone C (2016) Protecting cognition from aging and Alzheimer's disease: A computerized cognitive training combined with reminiscence therapy. Int J Geriatr Psychiatry 31, 340-348.

[75] Silverberg NB, Ryan LM, Carrillo MC, Sperling R, Petersen RC, Posner HB, Snyder PJ, Hilsabeck R, Gallagher M, Raber J, Rizzo A, Possin K, King J, Kaye J, Ott BR, Albert MS, Wagster MV, Schinka JA, Cullum CM, Farias ST, Balota D, Rao S, Loewenstein D, Budson AE, Brandt J, Manly JJ, Barnes L, Strutt A, Gollan TH, Ganguli M, Babcock D, Litvan I, Kramer JH, Ferman TJ (2011) Assessment of cognition in early dementia. Alzheimers Dement 7, e60-e76. 